\title{
GATE simulation of a new design of pinhole SPECT system for small animal brain imaging
}

\author{
Dilber Uzun Ozsahin ${ }^{\mathrm{a}, \mathrm{b}^{*}}$, Lisa Bläckberg ${ }^{\mathrm{a}, \mathrm{c}}$, Georges El Fakhri ${ }^{\mathrm{a}}$, Hamid Sabet ${ }^{\mathrm{a}}$ \\ ${ }^{a}$ Gordon Center for Medical Imaging, Department of Radiology, Massachusetts General Hospital and \\ Harvard Medical School, Boston, USA \\ ${ }^{b}$ Near East University, Department of Biomedical Engineering, Nicosia, Cyprus \\ ${ }^{c}$ Department of Physics and Astronomy, Uppsala University, Sweden \\ E-mail: dilber.uzunozsahin@neu.edu.tr, hsabet@mgh.harvard.edu
}

\begin{abstract}
Small animal SPECT imaging has gained an increased interest over the past decade since it is an excellent tool for developing new drugs and tracers. Therefore, there is a huge effort on the development of cost-effective SPECT detectors with high capabilities. The aim of this study is to simulate the performance characteristics of new designs for a cost effective, stationary SPECT system dedicated to small animal imaging with a focus on mice brain. The conceptual design of this SPECT system platform, Stationary Small Animal SSA-SPECT, is to use many pixelated CsI:TI detector modules with $0.4 \mathrm{~mm}$ x $0.4 \mathrm{~mm}$ pixels in order to achieve excellent intrinsic detector resolution where each module is backed by a single pinhole collimator with $0.3 \mathrm{~mm}$ hole diameter. In this work, we present the simulation results of four variations of the SSA-SPECT platform where the number of detector modules and FOV size is varied while keeping the detector size and collimator hole size constant. Using the NEMA NU-4 protocol, we performed spatial resolution, sensitivity, image quality simulations followed by a Derenzo-like phantom evaluation. The results suggest that all four SSA-SPECT systems can provide better than $0.063 \%$ system sensitivity and $<1.5 \mathrm{~mm}$ FWHM spatial resolution without resolution recovery or other correction techniques. Specifically, SSA-SPECT-1 showed a system sensitivity of $0.09 \%$ in combination with $1 \mathrm{~mm}$ FWHM spatial resolution.
\end{abstract}

KEYWORDS: Small animal SPECT, GATE, MLEM, CsI:T1 scintillator, laser processing, pinhole collimator 


\section{Contents}

1. Introduction $\quad 1$

$\begin{array}{ll}1.1 \text { System Specifications } & 2\end{array}$

2. Performance Evaluation $\quad 3$

2.1 System Sensitivity and Spatial Resolution 3

2.2
NEMA Image Quality Phantom

2.3 Derenzo-like Phantom Study $\quad 5$

$\begin{array}{lr}\text { 3. Conclusion } & 6\end{array}$

\section{Introduction}

Small animal SPECT imaging has gained tremendous attention in both clinical and research applications. This is due to the fact that it can help discovering new drugs, tracers, and therapeutic regimens [1]. In this regard, semiconductor detectors such as cadmium telluride (CdTe) and cadmium zinc telluride (CZT) have gained much interest for SPECT systems in general, and for small animal SPECT in particular, mainly due to their superior energy resolution. However, the main drawback associated with these semiconductor detectors is their high fabrication cost [2]. Scintillator crystals, on the other hand, have been the gold standard in nuclear imaging systems since the introduction of the Anger camera due to their decent stopping power, and relatively low price [3]. One main reason that semiconductors are more favorable than scintillators for small animal SPECT detectors is the high precision segmentation (pixelation) technology for semiconductors that can provide pixelated detectors with very small pixel size with high precision. The pixelation technology has been one of the bottlenecks of using scintillators when detectors with small pixel sizes are required. Conventional mechanical pixelation techniques are labor-intensive and cost-prohibitive, especially for fabrication of sub-mm pixels that are of interest for small animal imaging. With the increasing demand for higher performance small animal SPECT systems, many research groups have been developing new detector designs that can faithfully provide both high spatial resolution and high sensitivity in a cost effective manner [4-9].

In this work we report on simulation results for a new design for a mouse brain SPECT system which is based on finely segmented CsI:Tl scintillators which provide high intrinsic detector resolution (IDR) while maintaining a high sensitivity to the incident gamma rays. The enabling technique for cost-effective fabrication of such detectors is the emerging technique called Laser Induced Optical Barriers (LIOB), which can be used as an alternative to mechanical pixelation methods [10-15]. A high IDR detector allows for a shorter detector to collimator distance while maintaining a high system spatial resolution. Therefore, more detectors can be packed around the object (e.g. small animal), which leads to increased sensitivity and larger number of sampling views. Here we present the performance evaluation of our proposed SSASPECT system using the GATE simulation toolkit [16]. We have studied the spatial resolution 
and system sensitivity of four variations of the SSA-SPECT platform, followed by phantom studies of one selected design.

\subsection{System Specifications}

The SSA-SPECT platform is based on using multi detector-collimator pairs where each pair works as a complete gamma camera and sees the entire FOV. By placing many of these detectorcollimator pairs around the object, one can expect to achieve high system sensitivity and large number of view angles in a stationary configuration. Figure 1 shows the geometry of one SSASPECT-1 detector module. It contains a $17.4 \times 17.4 \times 5 \mathrm{~mm}^{3}$ CsI:Tl crystal pixelated into a $0.4 \mathrm{x}$ $0.4 \mathrm{~mm}^{2}$ pixel array. The scintillator array is optically coupled to an $8 \times 8$ array of Multi-Pixel Photon Counters (MPPC) with the same cross-sectional size. Each such detector module is backed by a single pinhole collimator with $0.3 \mathrm{~mm}$ hole diameter (Figure 1). The four variations of our design differ in the collimator to detector distance, as well as the collimator to center of FOV distance, and hence the magnification factor. The size of the detector is kept the same in all four systems leading to different acceptance angles of the collimators. The details of the four systems are outlined in Table 1.

\section{$17.4 \mathrm{~mm}$}

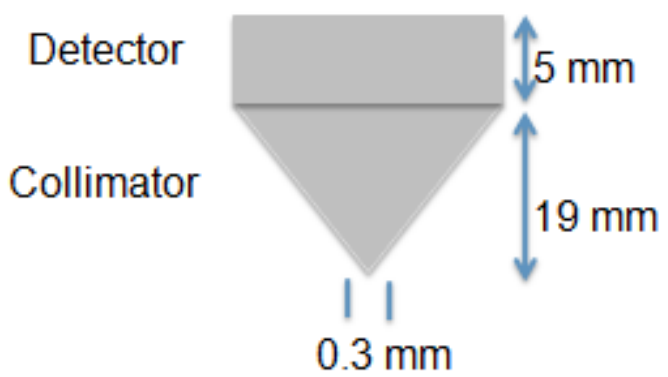

Figure 1. Side view of a single SSA-SPECT-1 detector module.

Figure 2 shows how the detector modules are arranged in three complete rings around the object. The lateral rings are slightly tilted to focus towards the field of view making this configuration especially useful for small animal brain imaging. It is known that large number of sampling views can result in noise reduction in the reconstructed image.
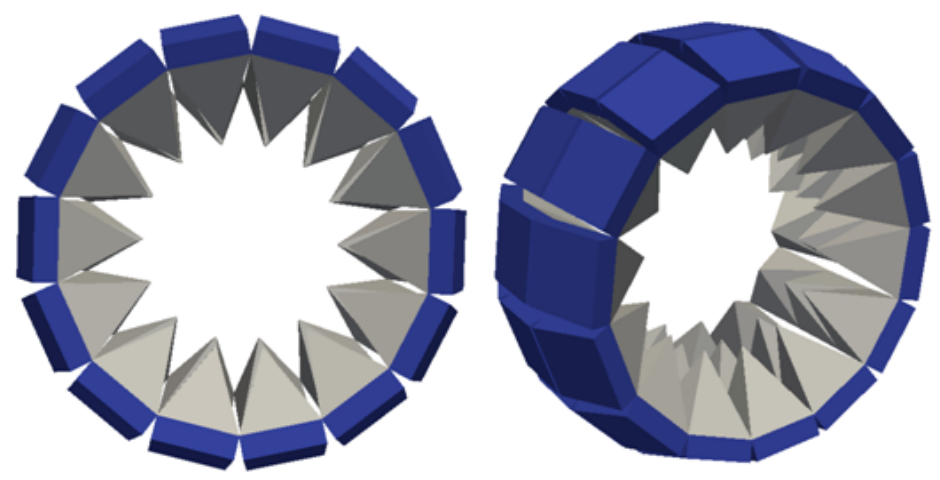

Figure 2. GATE render of the SSA-SPECT-1 system where 42 detector-collimator modules are arranged in three detector rings. 


\section{Performance Evaluation}

\subsection{System Sensitivity and Spatial Resolution}

We used NEMA Standards Publication NU4-2008 [17] to evaluate the system sensitivity and spatial resolution of the SPECT systems. Key parameters of all four designs are summarized in Table 1.

Following the NEMA protocol, we implemented a single point-like ${ }^{99 \mathrm{~m}} \mathrm{Tc}$ source $(0.15 \mathrm{~mm}$ in diameter) embedded in a $10 \times 10 \times 10 \mathrm{~mm}^{3}$ acrylic cube placed in the center of the FOV. For this test, we collected 100,000 singles events and used the Maximum Likelihood Expectation Maximization (MLEM) algorithm for image reconstruction.

Figure 3 shows the resulting system sensitivity and spatial resolution for each of the SSASPECT designs. We selected the SSA-SPECT-1 system for further evaluation since it provides the highest system sensitivity and smallest number of detector modules.

Table 1. System specifications of the studied small animal brain SPECT designs.

\begin{tabular}{|c|c|c|c|c|}
\hline & SSA-SPECT-1 & SSA-SPECT-2 & SSA-SPECT-3 & SSA-SPECT-4 \\
\hline Detector size $\left(\mathrm{mm}^{2}\right)$ & \multicolumn{5}{|c|}{0.4} \\
\hline Detector pixel pitch $(\mathrm{mm})$ & \multicolumn{5}{|c|}{0.3} \\
\hline Pinhole diameter $(\mathrm{mm})$ & 42.4 \\
\hline Number of detector modules & 42 & 45 & 48 & 45 \\
\hline Collimator focal length (mm) & 19 & 22 & 25 & 20 \\
\hline $\begin{array}{c}\text { FOV center to pinhole } \\
\text { distance (mm) }\end{array}$ & 25 & 27 & 28 & 30 \\
\hline Magnification & 0.76 & 0.81 & 0.89 & 0.66 \\
\hline FOV size (mm) & $\sim 23$ & $\sim 21$ & $\sim 19$ & $\sim 26$ \\
\hline
\end{tabular}

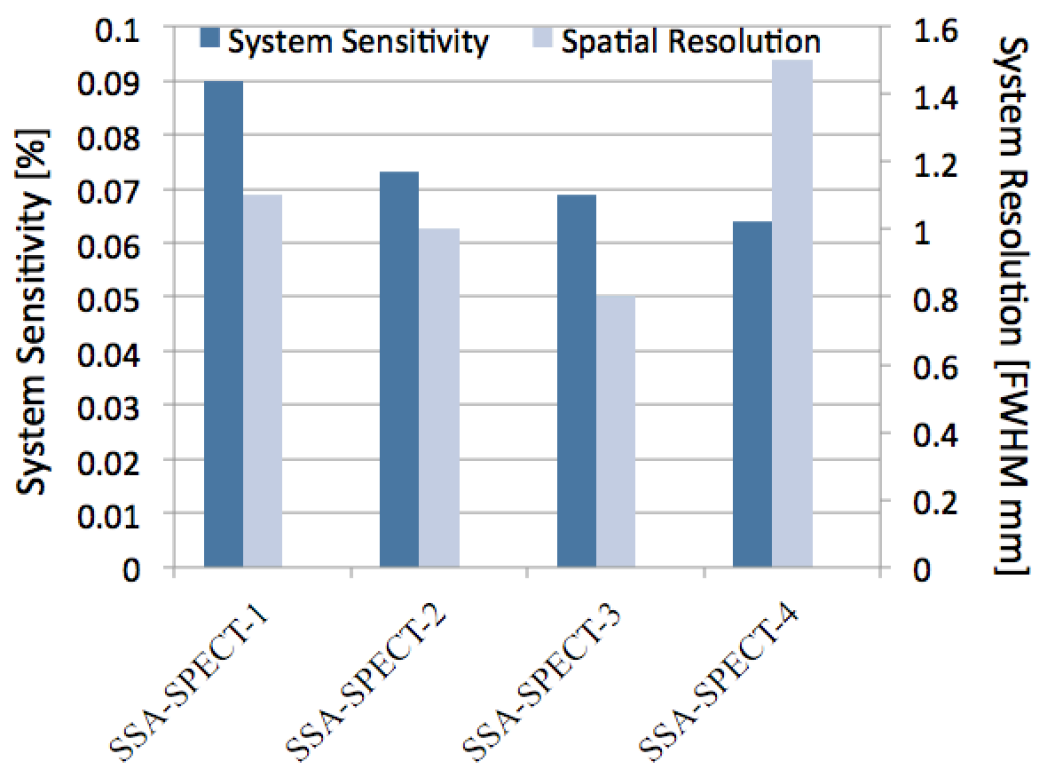

Figure 3. Spatial resolution and sensitivity results for the SSA-SPECT designs. No correction techniques were applied for any of the systems. 


\subsection{NEMA Image Quality Phantom}

To evaluate the imaging performance of the SSA-SPECT-1 system, we used a modified NEMA NU 4-2008 image quality phantom shown in Figure 4 [18]. It was necessary to modify the NEMA phantom such that it can fit in the FOV of the SSA-SPECT designs. The simulated phantom is made of polymethacrylate and has internal dimensions of $50 \mathrm{~mm}$ in length and $20 \mathrm{~mm}$ in diameter. The phantom consists of three parts: the fillable uniformity chamber ( $30 \mathrm{~mm}$ long, 20 $\mathrm{mm}$ diameter) enclosing two smaller, fillable cylinders (14 $\mathrm{mm}$ long, $5 \mathrm{~mm}$ outer diameter, $1 \mathrm{~mm}$ wall thickness), which are attached to the lid and serve as containers of cold matter (nonradioactive water and air). The third compartment consists of a solid cylinder ( $20 \mathrm{~mm}$ long, 20 $\mathrm{mm}$ diameter) containing 5 fillable rods at a $7 \mathrm{~mm}$ distance from the cylinder center. The rod diameters are 1, 2, 3, 4 and $5 \mathrm{~mm}$, respectively. The rods and the main body's chamber are filled with ${ }^{99 \mathrm{~m}} \mathrm{Tc}$ radioactive water with a total activity of $3.7 \mathrm{MBq}$ per NEMA NU-4's instruction. A total of 50 million singles were collected for each scan. The resulting images of the three regions, and the corresponding line profiles are shown in Figures 5 and 6.

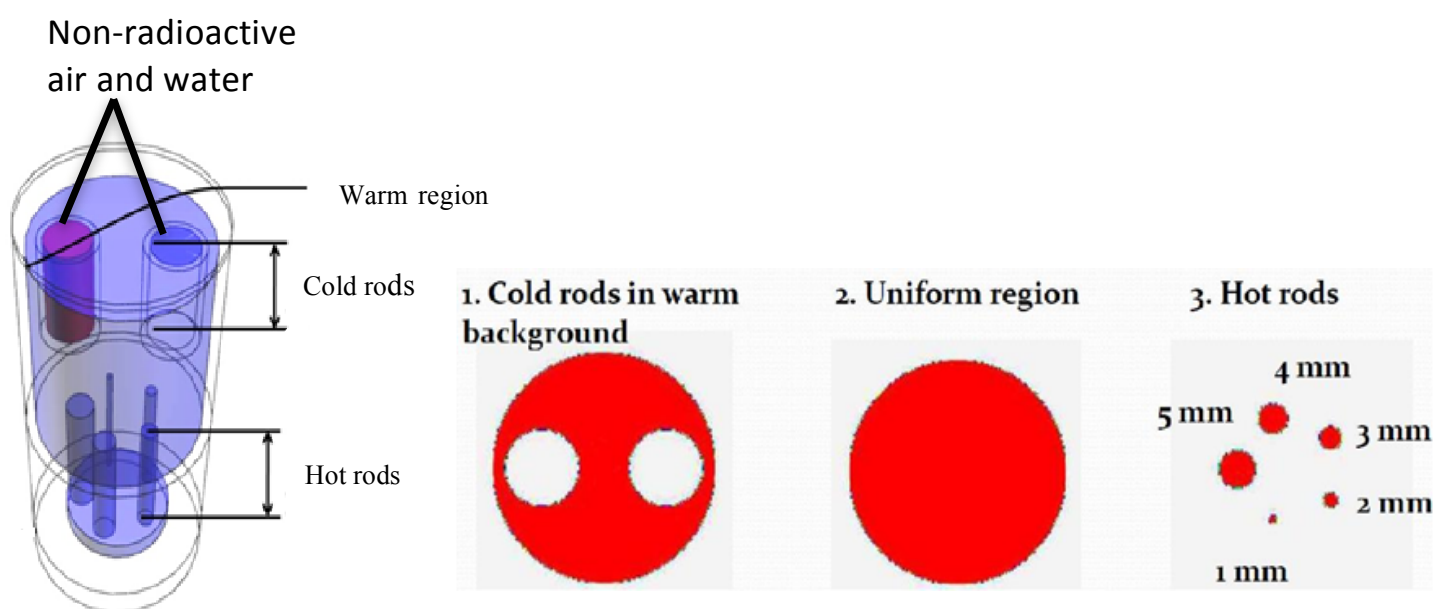

Figure 4. Left: A representation of a modified NEMA Image Quality phantom. Right: digital map representing the ideal images of the three sections of the phantom.
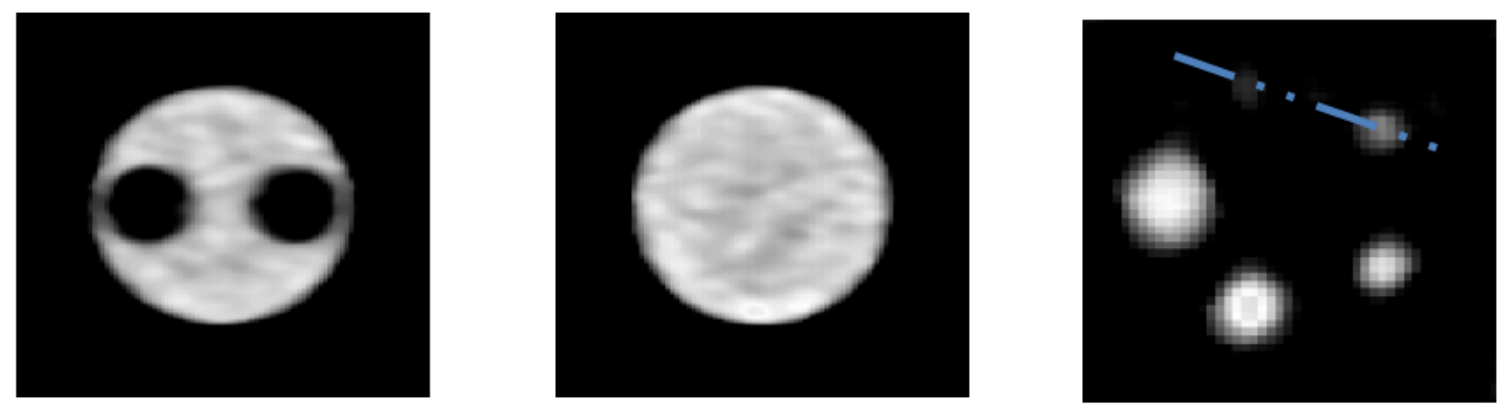

Figure 5. Images of the modified NEMA NU4-2008 phantom which is aligned to the center of the FOV of the SSA-SPECT-1 system. The images are reconstructed using the MLEM algorithm with 50 iterations. Left: cold inserts region; middle: uniform region; right: hot rods region. 

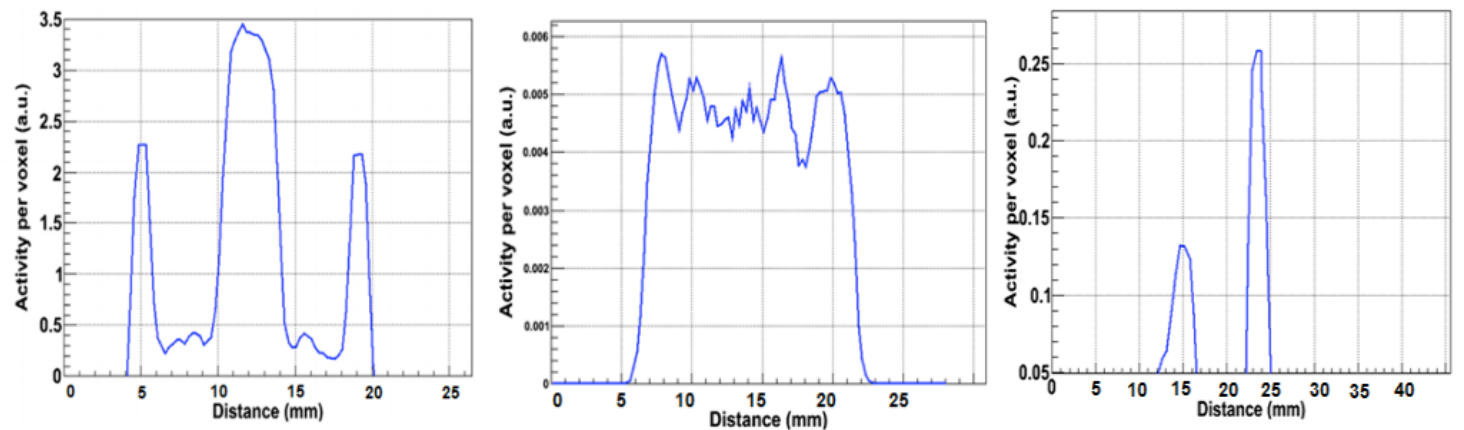

Figure 6. Corresponding line profiles from the phantom images in Figure 5. Left: cold inserts region; middle: uniform region; right: line profiles along the $1 \mathrm{~mm}$ and $2 \mathrm{~mm}$ hot rods.

Table 2. Recovery coefficients and standard deviation values of the NEMA image quality phantom.

\begin{tabular}{|c|c|c|c|c|c|}
\hline Rod Diameter & $1 \mathrm{~mm}$ & $2 \mathrm{~mm}$ & $3 \mathrm{~mm}$ & $4 \mathrm{~mm}$ & $5 \mathrm{~mm}$ \\
\hline RC & 0.35 & 0.48 & 0.57 & 0.64 & 0.85 \\
\hline \%STD & 36.5 & 25.2 & 17.9 & 13.1 & 11.5 \\
\hline
\end{tabular}

Calculations of the recovery coefficient $(\mathrm{RC})$ and the image uniformity were done with the phantom aligned with the center of the FOV. The uniform region shows good uniformity with $12.3 \%$ standard deviation (STD). Due to the partial volume effect the RC increases with bigger diameter rods and the STD of the RC decreases, as shown in Table 2.

\subsection{Derenzo-like Phantom Study}

In addition to the NEMA phantom, the imaging performance of SSA-SPECT-1 was evaluated using a Derenzo-like hot sphere phantom. This phantom has five sectors containing small hot spheres with diameters of $1,1.25,1.5,1.75$, and $2.5 \mathrm{~mm}$ as shown in Figure 7 . The spherical sources are placed in a $22 \mathrm{~mm}$ diameter air cylinder. Figure 7 also shows the reconstructed images of the phantom using the MLEM reconstruction algorithm with 60 iterations. As shown in the image and the corresponding line profile, $1 \mathrm{~mm}$ hot spheres can be resolved without applying any correction techniques such as resolution recovery.

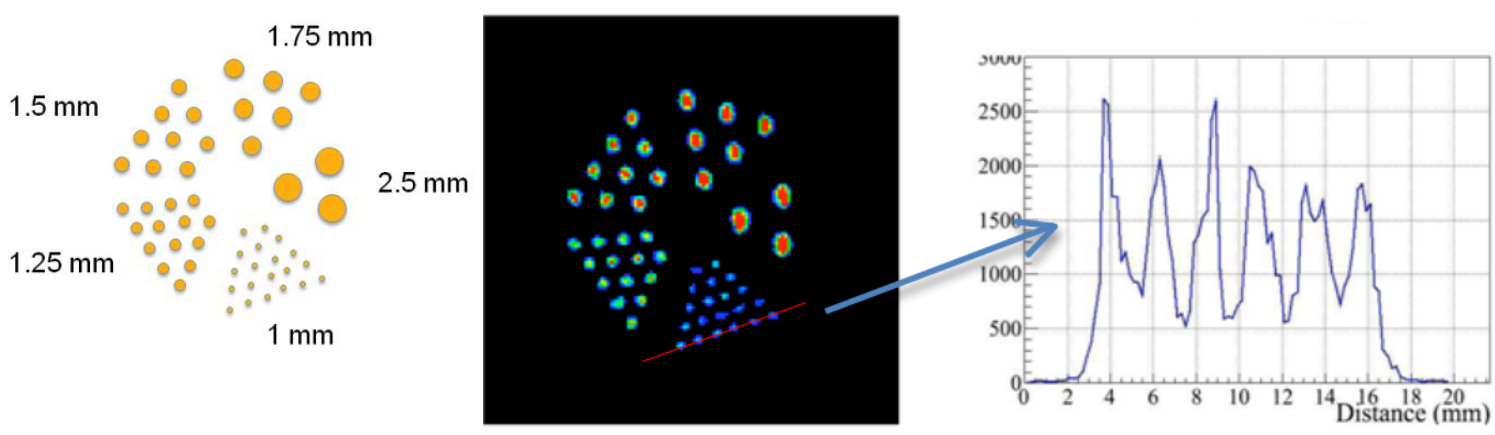

Figure 7. Left: A schematic view of the Derenzo-like phantom; middle: reconstructed image of the phantom placed in the FOV center of SSA-SPECT-1; right: line profile through the $1 \mathrm{~mm}$ diameter spheres. 


\section{Conclusion}

While it is apparent that using a high IDR detector is key to achieve high performance, system development cost should also be practical. Previously we reported that by using detector fabrication techniques such as LIOB, fabrication of high IDR detectors for small animal SPECT is feasible [14]. Combining those experimental results with the preliminary simulation results presented here is encouraging as it shows that the SSA-SPECT platform can potentially provide both high sensitivity and high spatial resolution for small animal brain imaging in a practical manner. Through the simulation results, we have shown that SSA-SPECT-1 can achieve $\sim 1 \mathrm{~mm}$ FWHM spatial resolution and $\sim 0.09 \%$ sensitivity with no rotating components such as collimators or detectors, and no translation of the animal bed. The Derenzo-like phantom image also confirms that with this system, the $1 \mathrm{~mm}$ diameter spheres are well separated. Noteworthy is that this performance is achieved with no system modeling applied.

\section{Acknowledgments}

This work was supported in part by the US National Institute of Health under Grant No. 1R03EB020762-01 and 1R21EB020162-01A1. D.U.O. acknowledges support from the Scientific and Technological Research Council of Turkey (TUBITAK) and Center of Excellence, Near East University, L.B. acknowledges financial support from the Swedish Research Council (VR).

\section{References}

[1] B. S. Jang, MicroSPECT and MicroPET Imaging of Small Animals for Drug Development, Toxicol Res. 29 (2013) 1.

[2] M. T. Madsen, Recent advanced in SPECT imaging, J Nucl. Med. 48 (2007) 661.

[3] M. M. Khalil et al., Molecular SPECT Imaging: An Overview, International Journal of Molecular Imaging Article ID 796025 (2011).

[4] L. Cai et al., MRC-SPECT: A sub-500 um resolution MR-compatible SPECT system for simultaneous dual-modality study of small animals, Nucl. Inst. Phys. Res. A. 734(B) (2014) 147.

[5] B. W. Miller et al., Progress in Bazooka SPECT, Proc. SPIE, San Diego, U.S.A. September 2009.

[6] LC. Johnson et al., Characterization of a high-purity germanium detector for small-animal SPECT, Phys. Med. Biol. 56 (2011) 5877.

[7] T. E. Peterson et al., Multi-pinhole SPECT Imaging with Silicon Strip Detectors, IEEE Trans. Nucl. Sci. 56 (2009) 646.

[8] L. R. Furenlid et al., FastSPECTII: A Second-Generation High-Resolution Dynamic SPECT Imager, IEEE Trans. Nucl. Sci. 51 (2004) 631.

[9] R. Van Holen et al., Design and performance of a compact and stationary microSPECT system, Med Phys. 40 (2013) 112501.

[10] T. Moriya et al., Development of PET detectors using monolithic scintillation crystals processed with sub-surface laser engraving technique, IEEE Trans. Nucl. Sci. 57 (2010) 2455. 
[11] H. Sabet et al., Fabricating high resolution and high-sensitivity scintillator arrays using laser induced optical barriers, IEEE NSS-MIC Conf. Rec., Anaheim, U.S.A. October 2012.

[12] W. C. Hunter et al., Light-sharing interface for dMICE detectors using sub-surface laser engraving, IEEE Trans. Nucl. Sci. 62 (2015) 27.

[13] H. Sabet et al., A sub-mm spatial resolution LYSO:Ce detector for small animal PET, IEEE NSSMIC Conf. Rec., San Diego, U.S.A., October 2015.

[14] H. Sabet et al., Novel laser-processed CsI:Tl detector for SPECT, Med. Phys. 43 (2016) 2630.

[15] H. Ushida et al., A novel single-ended readout depth-of-interaction PET detector fabricated using sub-surface laser engraving, Phy. Med. Biol. 61 (2016) 6635.

[16] S. Jan et al., GATE: A simulation toolkit for PET and SPECT, Phys. Med. Biol. 49 (2004) 4543.

[17] NEMA Standards Publication NU 4-2008: Performance Measurements of Small Animal Positron Emission Tomographs, National Electrical Manufacturers Association 2008.

[18] E Mikhaylova et al., Optimization, evaluation, and comparison of standard algorithms for image reconstruction with the VIP-PET, J. Instrum. 9 (2014) C07004. 